\title{
Successive approximation method applying to obtain the transient elastic medium deformations
}

\author{
Volodymyr Sapehin ${ }^{1, *}$ \\ ${ }^{1}$ Institute of Geotechnical Mechanics named by N. Poljakov of National Academy of Sciences of \\ Ukraine, 49005, Dnipro, Simferopolska Str., 2a, Ukraine
}

\begin{abstract}
Therein used the well-known method of successive approximation of functions for solving the problem of transient deformation of an elastic medium under the influence of variable internal pressure in wells in paper. The desired function - the maximum tensile stresses - is represented as a product of power functions, each of which depends on only one parameter. The regularity of changes in the maximum tensile stresses and the main factors influencing this process, which is fully confirmed by the independent theory of dimensions, are established.
\end{abstract}

\section{Introduction}

In practice, the class of power functions [1] has become widespread for representing functional dependencies. The main advantages of such a presentation are the ability to assess the degree of influence of parameters on the function itself and determine the compliance of the physical content of the resulting dependencies with the properties of the original process function. In connection with the noted features, we will look for the representation of the original function as a product of power functions.

Using the method of successive approximation [1, 2], all numerical solutions were analyzed on the basis of the integral Fourier conversion, as well as solutions for the ratio of the external diameter of a hollow cylinder $b$ to internal $a$ equal to $10>b / a>1.1$ using model A.N. Krylov, for which the author of the work has developed the conditions for its use for the relation $\infty \geq b / a>1.1$ [3].

For the case of plane deformation, the calculation of radial $\sigma_{\mathrm{r}}$ and tangential $\sigma_{\theta}$ stresses can be carried out using the formulas given in [4].

The main parameters influencing the process of transient deformation of an elastic medium include the inner radius of the well $r_{0}$, the internal pressure $p_{0}$ of the working agent, the elastic wave velocity in the massif $v_{p}$, and the internal pressure in the working agent release time $t_{c}$.

All further calculations and transformations are reduced to the maximum value of tensile radial stresses in the first half-wave of stress variation at the site of inertial forces (Fig. 1) [5].

*Corresponding author: vladimirsapegin10@gmail.com 


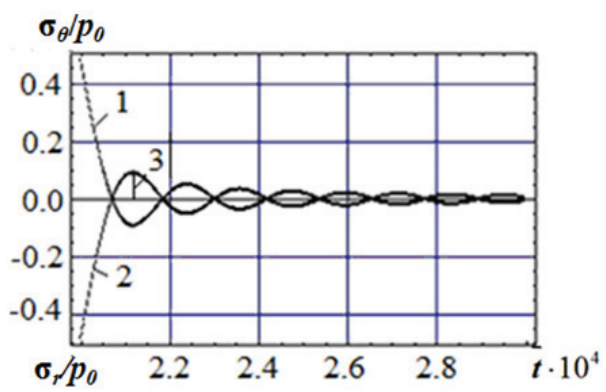

Fig. 1. Change of radial and tangential stresses in time in dimensionless form at the section of inertial force action when the internal pressure is released from the well: 1 - tangential stress; 2 - radial stress; 3 - maximum tensile stresses in the first half-wave.

\section{Problem definition and solution method}

Let us present the solution of this problem in the form of simple engineering formulas convenient for calculating stresses or displacements of an elastic medium in the vicinity of wells during short-term unloading using the method of successive approximation of a variable $[1,2]$.

The essence of the method is that the resultant function, according to the results of its numerical research and given in tabular form, can be presented in an analytical form - a product of functions, each of which depends only on one variable.

Let in general, the form of the solution is represented as a function $F(x)=F\left(x_{1}, x_{2}, \ldots, x_{n}\right)$ defined and continuous in a closed area $D$. In a neighborhood of a point $X_{0}=\left(x_{1}{ }^{0}, x_{2}{ }^{0}, \ldots\right.$, $\left.x_{n}{ }^{0}\right) \in D$ function $F(x)$ can be represented as a dependence given in [1].

$$
F(X) \approx \varphi\left(x_{1}, \ldots, x_{n}\right)=c \prod_{i=1}^{n} g_{i}\left(x_{i}\right)
$$

where $g_{i}\left(x_{i}\right)$ - approximation functions for $f_{1}, f_{2}, \ldots, f_{n}$ specified in tabular form; $c$ proportionality coefficient.

Let's select reference point $M=M\left(x_{1}{ }^{0}, x_{2}{ }^{0}, \ldots, x_{n}{ }^{0}\right), M \in D$, where $x_{1}{ }^{0}, x_{2}{ }^{0}, \ldots, x_{n}{ }^{0}-$ average values of the studied parameters in a given range of their changes.

These functions $g_{i}\left(x_{i}\right)$ are formed as follows: we fix the average values of the studied parameters and vary only one of them in the range of its change, then establish the type of function and the exponent of the degree of its influence $\alpha_{i}: f_{1}\left(x_{1}\right)=F\left(x_{1}, x_{2}{ }^{0}, \ldots, x_{n}{ }^{0}\right)$; $f_{2}\left(x_{2}\right)=F\left(x_{1}^{0}, x_{2}, \ldots, x_{n}{ }^{0}\right) ; f_{n}\left(x_{n}\right)=F\left(x_{1}{ }^{0}, x_{2}{ }^{0}, \ldots, x_{n}\right)$.

Repeating this approximation procedure successively for each parameter studied $\left(x_{1}\right.$, $x_{2}, \ldots, x_{n}$ ), we obtain the desired representation in the form:

$$
F\left(x_{1}, x_{2}, x_{3}, \ldots, x_{n}\right) \approx \varphi\left(x_{1}, x_{2}, x_{3}, \ldots, x_{n}\right)=c_{n} \cdot g_{1}\left(x_{1}\right) \cdot g_{2}\left(x_{2}\right), \ldots, \cdot g_{n}\left(x_{n}\right) .
$$

The total proportionality coefficient for all parameters is determined by the formula:

$$
c_{n}=F\left(x_{1}{ }^{0}, x_{2}{ }^{0}, \ldots, x_{n}{ }^{0}\right) / g_{1}\left(x_{1}{ }^{0}\right)^{\alpha 1} \cdot g_{2}\left(x_{2}{ }^{0}\right)^{\alpha 2}, \ldots, \cdot g_{n}\left(x_{n}{ }^{0}\right)^{\alpha \mathrm{n}} .
$$

\section{Results and discussion}

As a function, which we are looking for, we take the value of the maximum tensile stresses in the first half-wave of its change, referred to the maximum amplitude of the internal pressure $p_{0}$ before the relief. 


$$
\sigma_{r} / p_{0}=\sigma\left(t_{c}^{\alpha 1} \cdot v_{p}^{\alpha 2} \cdot r_{0}^{\alpha 3}\right)
$$

where $\alpha_{1}, \alpha_{2}, \alpha_{3}$ - exponents of the functions of the parameters studied.

The domain of definition is defined by the intervals of change of all the main parameters: the interval of change of the inner radius of the cylindrical cavity $r_{0}$ equals 0.05 - $0.5 \mathrm{~m}$; elastic wave velocity $v_{p}$ equals $500-2500 \mathrm{~m} / \mathrm{s}$; time of pressure relief $t_{c}$ equals $0.001-0.5 \mathrm{~s}$.

Reference point $M=M\left(x_{1}{ }^{0}, x_{2}{ }^{0}, \ldots, x_{n}{ }^{0}\right), M \in D$, is set by average values of the parameters: pressure release time $t_{c}{ }^{0}=0.01 \mathrm{~s}$ elastic wave velocity $v_{p}{ }^{0}=600 \mathrm{~m} / \mathrm{s}$, borehole radius $r_{0}^{0}=0.1 \mathrm{~m}$.

Changing a parameter $t_{c}$ with a certain step, we calculate the sequence of function $\sigma_{r} / p_{0}$ values. For the sequence $\left(t_{c}, \sigma_{r} / p_{0}\right)$ we will find approximation function $g_{1}\left(t_{c}\right)$ in the form: $g_{1}\left(t_{c}\right)=c_{1} t_{c}^{\alpha 1}$, where: $c_{1}=0.0002018 ; \alpha_{1}=-0.96$.

Graphical representation of the implementation of these stages is presented in Figure 2.

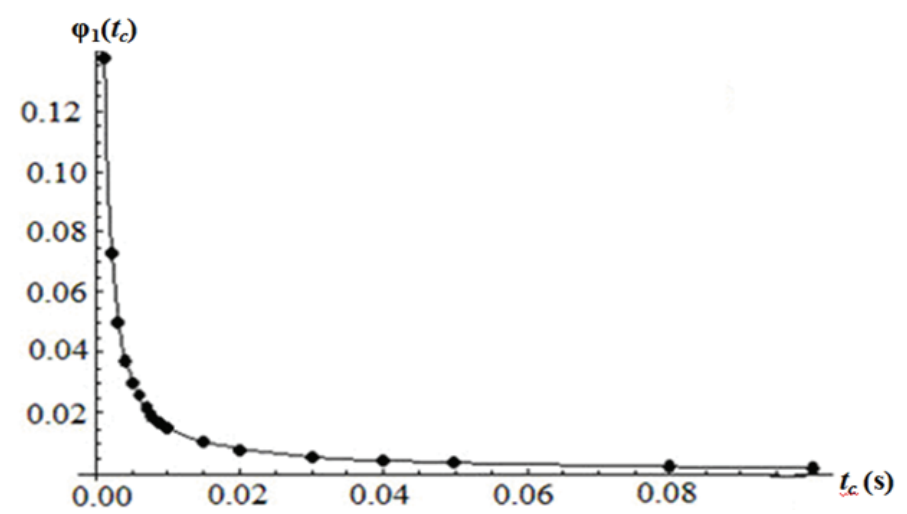

Fig. 2. Function $\varphi_{1}\left(t_{c}\right)$ in tabular form and its approximation function.

Thus, the dimensionless tensile stresses load release time $t_{c}$ will have the form:

$$
\sigma_{r} / p_{0}\left(t_{c}, v_{p}{ }^{0}, r_{0}{ }^{0}\right) \approx \varphi_{1}\left(t_{c}\right)=c_{1} \cdot t_{c}^{\alpha 1}
$$

Formula (5) can already be used in calculations to determine tensile stresses depending on the different release times (for fixed values of the parameters at the reference points $v_{p}{ }^{0}$ and $r_{0}{ }^{0}$.

Changing the next parameter $v_{p}$, with a certain step, we calculate the sequence of function values $\sigma_{r} / p_{0}$. For sequence $\left(v_{p}, \sigma_{r} / p_{0}\right)$ we will find approximation function $g_{2}\left(v_{p}\right)$ in the form:

$$
g_{2}\left(v_{p}\right)=c_{2} \cdot v_{p}^{\alpha 2}
$$

where $c_{2}=0.09132 ; \alpha_{2}=-0.957$.

Graphical representation of the implementation of these stages is presented in Figure 3.

Thus, the dimensionless tensile stresses from the time of pressure drop $t_{c}$, and the elastic wave velocity $v_{p}$ will have the form

$$
\sigma_{r} / p_{0}\left(t_{c}^{0}, v_{p}, r_{0}^{0}\right) \approx \varphi_{2}\left(t_{c}, v_{p}\right)=c_{2} \cdot t_{c}^{\alpha 1} \cdot v_{p}^{\alpha 2} .
$$

Formula (6) can be used for particular calculations of stresses with a fixed value of the inner radius $r_{0}{ }^{0}$ and time $t_{c}{ }^{0}$. 


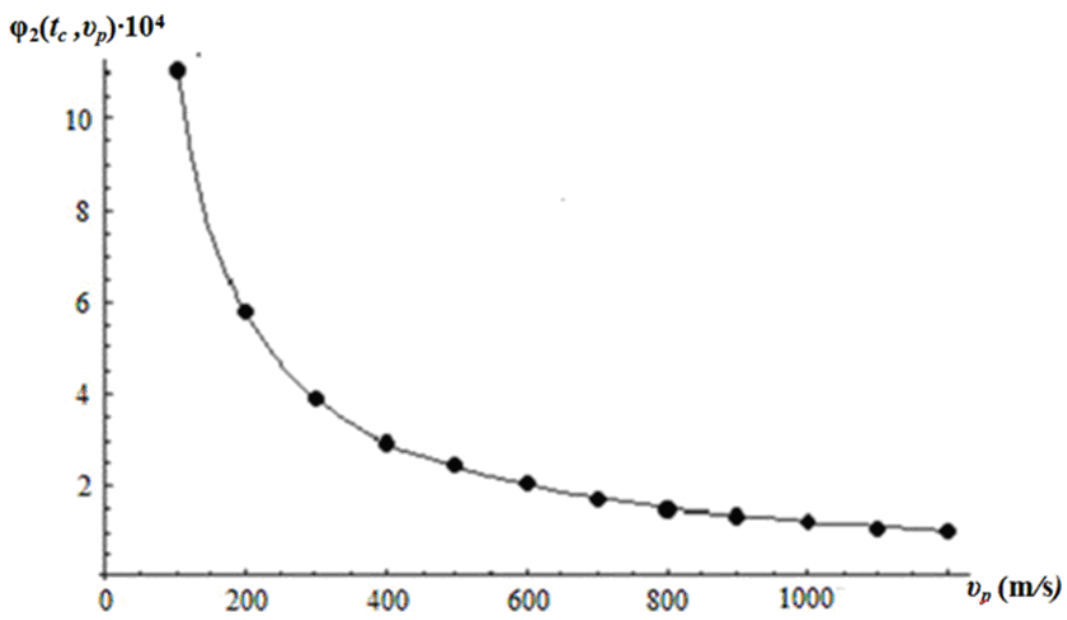

Fig. 3. Function $g_{2}\left(v_{p}\right)$ in tabular form and its approximation function.

Doing so and changing the parameter $r_{0}$ with a certain step, we calculate the sequence of values of the function $\sigma_{r} / p_{0}$. For sequence $\left(r_{0}, \sigma_{r} / p_{0}\right)$ we will find approximation function $g_{3}\left(r_{0}\right)$ in the form:

$$
g_{3}\left(r_{0}\right)=c_{3} r_{0}^{\alpha 3}
$$

where $c_{3}=0.9172 ; \alpha_{3}=1.01$.

Graphical representation of the implementation of these stages is presented in Figure 4.

Thus, the dimensionless tensile stresses from the load release time $t_{c}$ and the velocity of the elastic wave $v_{p}$ and the inner radius $r_{0}$ of the well will have the form:

$$
\sigma_{r} / p_{0}\left(t_{c}{ }^{0} \cdot v_{p}{ }^{0}, r_{0}\right) \approx \varphi_{3}\left(t_{c}, v_{p}, r_{0}\right)=c_{3} \cdot t_{c}^{\alpha 1} \cdot v_{p}{ }^{\alpha 2} \cdot r_{0}^{\alpha 3} .
$$

The final formula (8) for tensile radial stresses from all studied parameters is written in the form:

$$
\sigma_{r}=0.96 \cdot\left(p_{0}{ }^{1} \cdot r_{0}{ }^{1.01} / v_{p}{ }^{0.957} \cdot t_{c}^{0.96}\right) .
$$

Taking into account the proximity of all exponents of the studied parameters to a unit in expression (8), we obtain a more convenient linearized formula and regularity for calculating tensile stresses on the internal contour of the cylindrical cavity in the first halfwave of the variation of the stresses in the form:

$$
\sigma_{r}=p_{0} r_{0} / v_{p} \cdot t_{c}
$$

Comparison of the relative error of stresses calculated by formula (9) with the exact one, obtained by the method of the integral Fourier conversion and the method based on the solution of A.N. Krylov [3], showed that the average relative error for the release time does not exceed $5.7 \%$, for the elastic wave velocity $-5.2 \%$, and for the inner radius $-10 \%$.

The correctness of the physical content of the obtained solution (9) is easy to confirm on the basis of the theory of dimensions. According to the theory of dimensions, the unknown value of radial stresses on the inner radius of the cylindrical cavity of a well can be represented in the form of the product of power functions [6]:

$$
\sigma_{r}=b_{1} \cdot p_{0}{ }^{p} \cdot r_{0}{ }^{q} v_{p}{ }^{r} \cdot t_{c}{ }^{k},
$$

where $p, q, r, k$ - unknown exponents; $b_{1}$ - dimensionless proportionality coefficient. 


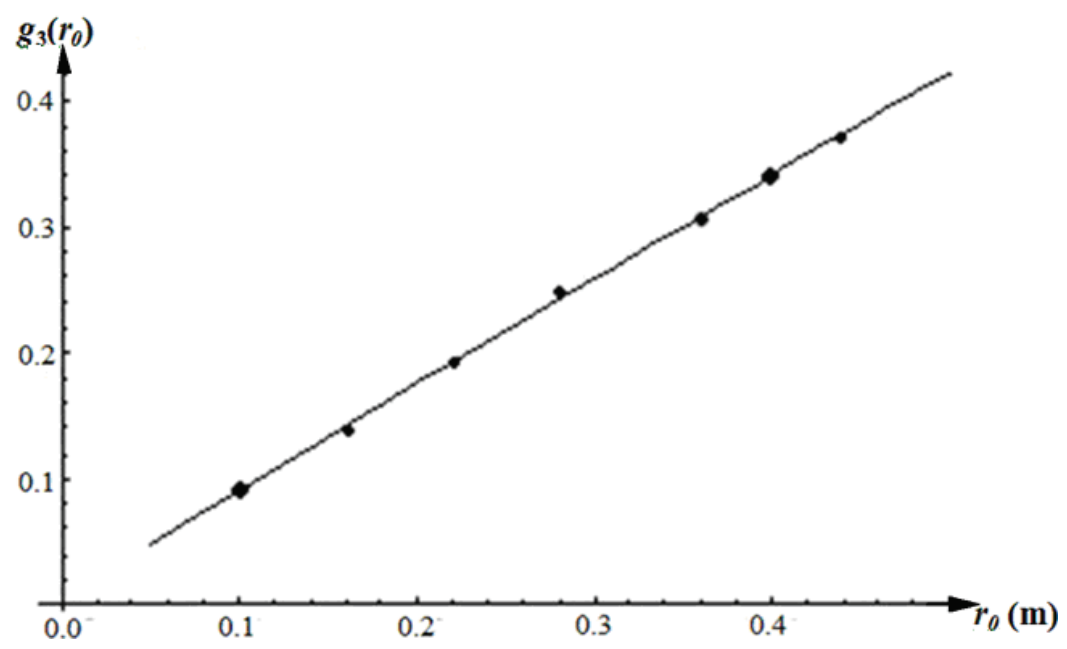

Fig. 4. Function $g_{3}\left(r_{0}\right)$ in tabular form and its approximation function.

Equating the dimensions on the left and right side of equation (10) we obtain:

$$
M \cdot T^{2} \cdot L^{-1}=M^{p} \cdot T^{2 p} \cdot L^{-p} \cdot L^{q} \cdot L^{r} \cdot T^{r} \cdot T^{k} .
$$

We construct the following equations to determine the exponents:

$$
\begin{array}{r}
p=1, \\
-2=-2 p-r+k, \\
-1=-p+q+r .
\end{array}
$$

We have three equations $(12,13,14)$ and four unknown exponents to be determined. From equation (12) we immediately obtain the exponent at $p_{0}$ equal to unit $p=1$. From equation (13) it follows that exponent at $v_{p}$ and $t_{c}$ equal to $r=k$. The exponent of influence of the inner radius $r_{0}$ is taken equal to the unit $q=1$ of the calculations given in [5].

Consequently, the remaining exponents from equation (14) with $v_{p}$ and $t_{c}$ will be equal to $r=k=-1$.

Thus, the general structure of formula (9) is fully confirmed by the independent theory of dimensionality, has the nature of regularities for linearly elastic media and is valid in a wide range of variation of the investigated variable parameters.

However, it should be noted that the theory of dimension does not always make it possible to find the proportionality coefficient $b_{1}$, connecting all variables. When using the method of successive approximation, it is found in a relatively simple way with any number of parameters.

The verification of the errors of approximate representations of functions specified in an analytical form using the method of successive approximation confirmed high accuracy for engineering calculations in the vicinity of the selected point $M\left(x_{1}{ }^{m}, x_{2}{ }^{m}, \ldots, x_{n}{ }^{m}\right)$ and good one throughout the entire domain.

\section{Conclusions}

The inertial tensile stresses that occur in the near-wellbore zone of the coal and rock massif after the working agent pressure release from wells, are directly proportional to its pressure before release and the well radius and inversely proportional to the velocity of the elastic 
wave in the massif and the time of pressure drop in the working agent. The time of pressure drop in the working agent is calculated in each case separately for a specific type of working agent and parameters of technological wells.

A rational value of the well radius for initiating the destruction of the carbon-forming medium is determined from the technological point of view and from the point of view of the fracture efficiency.

The speed of the elastic wave in the investigated disturbed coal-rock massif is determined by taking into account the degree of decrease in the elastic modulus during gas saturation.

The generality and reliability of the obtained regularity is confirmed by use of fundamental provisions of continuum mechanics, known classical solutions by Lame and Krylov, mine research by Institute of Geotechnical Mechanics named by N. Poljakov of National Academy of Sciences of Ukraine under hydrodynamic effects on gas-saturated coal beds. For the first time, a regularity has been established that relates the loading parameters, the elastic and deformation properties of the carbon-bearing medium, and the geometric parameters of the well.

For the first time, a regular approximation method is established for tensile stresses in the near-wellbore zone of a coal-bearing massif from the influence of inertial forces under the action of alternating internal pressure, which relates the pressure of the working agent in the well, the phase state and the time of pressure drop in the working agent, elastic and deformation parameters of the medium and geometric characteristics of well and developed on its basis a calculation method that allows you to determine the necessary values of loading parameters for coal media destruction initialization.

Application of the method of successive approximation to transform a complex solution of a dynamic problem allowed us to obtain a simple formula connecting the elastic characteristics of the massif, the geometric parameters of the elastic medium and the loading parameters. Using the solution obtained, it is easy to play different situations when internal pressure is released from wells, varying parameters in the range of their changes to obtain the desired value of tensile stresses. The application of the method of successive approximation to the analysis of the solution of the problem of transient deformation of an elastic medium under the action of a variable internal load made it possible for the first time to establish the pattern of change in inertial maximum radial tensile stresses.

\section{References}

1. Larionov, G.I. (2010). To the analysis of the results of numerical simulation. Materials of the International Scientific Conference "Mathematical Problems of Technical Mechanics", Dniprodzerzhinsk, April 19-22, 153

2. Larionov, G.I. (2010). Estimation of influence of parameters of mathematical modeling. Materials of the XVIII International Scientific and Technical Conference "Applied Problems of Mathematics and Mechanics". Sevastopol: SevNTU Publishing House, September 13-17, 226-229

3. Sapegin, V.N. (2012). Conditions for the use of the wave model A.N. Krylov for the calculation of elastic cylinders with large external boundaries under the action of a variable internal load. System Technologies. Region. mezhvuz. Sat scientific tr., 3 (80), 140 - 144

4. Lurie, A.I. (1970). Theory of elasticity. Moskva: Nauka

5. Sapegin, V.N. (2011). Unsteady elastic deformation among under the action of a variable internal load. Problems of calculating mechanics and tactical constructions, 17, 239-245

6. Sedov, L.I. (1977). Methods of similarity and dimension in mechanics. Moskva: Nauka 